Received: 10 May 2018

Accepted: 30 November 2018

Published online: 17 January 2019

\section{Identification of selective inhibitors of Helicobacter pylori IMPDH as a targeted therapy for the infection}

Kapil Juvale ${ }^{1,4}$, Gayathri Purushothaman ${ }^{2}$, Vijay Singh ${ }^{2}$, Althaf Shaik ${ }^{1}$, Srimadhavi Ravi ${ }^{1}$, Vijay Thiruvenkatam ${ }^{2,3}$ \& Sivapriya Kirubakaran ${ }^{1,2}$

Helicobacter pylori (H. pylori), the major cause of several gastric disorders has been recognied as a type I carcinogen. By virtue of resistance developed by $H$. pylori strains, currently used antibiotic based treatments rather demonstrate high failure rates. Hence, there is an emerging need for identification of new targets to treat $H$. pylori infection. Inosine- $5^{\prime}$-monophosphate dehydrogenase (IMPDH) has been studied as a potential target to treat $H$. pylori infection. Here, a detailed enzyme kinetic study of recombinant expressed $H$. pylori inosine- $5^{\prime}$-monophosphate dehydrogenase $(H p I M P D H)$ is presented. A new in-house synthesized indole-based scaffold is identified as an inhibitor for $\mathrm{HpIMPDH}$. These indole-based compounds showed non-competitive inhibition against IMP and NAD ${ }^{+}$whereas the benzimidazole compounds were found be uncompetitive inhibitors. The new indole scaffold ensures specificity due to its high selectivity for bacterial IMPDH over human IMPDH II. Our work aims to overcome the drawback of existing inhibitors by introducing new indole scaffold for targeting bacterial IMPDH.

Worldwide more than $50 \%$ population is infected by Helicobacter pylori (H. pylori), a gram-negative bacterium which resides in the antral region of stomach ${ }^{1}$. The infection rate is much higher (up to $80 \%$ ) in developing countries. Due to the lack of hygiene and sanitation, in India alone more than 20 million people are estimated to be suffering from gastric ulcer ${ }^{2}$. Although $H$. pylori do not show symptoms in most of the population, it has been associated with various disorders including gastric and duodenal ulcers, gastric atrophy and gastritis ${ }^{3}$. $H$. pylori is categorized as type I carcinogen due to its involvement in the pathology of gastric adenocarcinoma and malt associated lymphoid tissue lymphoma (MALT lymphoma) ${ }^{4}$. Currently, H. pylori infection is treated by either first line clarithromycin-based triple therapy or a quadruple therapy replacing clarithromycin by new antibiotics ${ }^{5}$. Despite these efforts, there is an increase in the antibiotic resistance shown by various strains of $H$. pylori. Hence, there is an emerging need to identify new targets to treat $H$. pylori infection, especially in developing countries like India.

Inosine-5'-monophosphate dehydrogenase (IMPDH, guaB, EC:1.1.1.205) has been demonstrated to be a promising target for several diseases. IMPDH is reported as a potential target for treating some pathogens like Cryptosporidium parvum (C. parvum) and Mycobacterium tuberculosis (M. tuberculosis) ${ }^{6,7}$. In the de novo biosynthesis of purine nucleotides, IMPDH enzyme is responsible for the oxidation of inosine $5^{\prime}$-monophosphate (IMP) to xanthosine $5^{\prime}$-monophosphate (XMP). The XMP is then further converted into guanosine $5^{\prime}$-monophosphate (GMP) by GMP synthase. Successive actions of several enzymes on GMP gives rise to some of the building blocks of DNA (dGTP) and RNA (dGTP) ${ }^{8,9}$. Hence, inhibition of IMPDH can stop the expansion of the guanine nucleotide pool that is needed for the microbial proliferation.

The phylogenetic analysis of $H$. pylori IMPDH (HpIMPDH) revealed its close resemblance to the C. parvum IMPDH (CPIMPDH) with a sequence identity of $60 \%$. This fact justifies the investigation of already available bacterial IMPDH inhibitors for HPIMPDH. Moreover, this similarity enables the designing of new inhibitors by in silico docking studies. Due to fundamental differences in the structural and kinetic properties of prokaryotic

${ }^{1}$ Chemistry, Indian Institute of Technology Gandhinagar, Palaj, Gandhinagar, 382355, India. ${ }^{2}$ Biological engineering, Indian Institute of Technology Gandhinagar, Palaj, Gandhinagar, 382355, India. ${ }^{3}$ Physics, Indian Institute of Technology Gandhinagar, Palaj, Gandhinagar, 382355, India. ${ }^{4}$ Present address: Shobhaben Pratapbhai Patel School of Pharmacy \& Technology Management, SVKM's NMIMS, V.L. Mehta Road, Vile Parle (W), Mumbai, 400056, India. Kapil Juvale and Gayathri Purushothaman contributed equally. Correspondence and requests for materials should be addressed to S.K. (email: priyak@iitgn.ac.in) 
<smiles>O=C(Cn1c(-c2ccccn2)nc2ccccc21)Nc1ccc2ccccc2c1</smiles>

1<smiles>O=C(Cn1cc(C(=O)Nc2cccnc2)c2ccccc21)Nc1ccc(Br)cc1</smiles>

2

Figure 1. Structures of reported benzimidazole (1,C91) and synthesised indole (2) based small molecules selected for the study.

IMPDH (Pro-IMPDHs) and eukaryotic IMPDH (Euk-IMPDHs), it is possible to develop selective inhibitors of bacterial enzyme ${ }^{10}$.

Gollapalli et al. have reported several inhibitors of CPIMPDH having selectivity over host (human) IMPDH II. It was shown that the benzimidazole class of small molecules are the most promising inhibitors of bacterial IMPDH. These compounds were found to bind in the cofactor $\left(\mathrm{NAD}^{+}\right)$binding pocket with one of the aromatic rings having $\pi-\pi$ interactions with $\mathrm{IMP}^{6,11,12}$. Even in eukaryotes, the IMP binding site of bacterial IMPDH enzyme is highly conserved. Hence, inhibitors binding to the IMP binding site can also inhibit the host (human) IMPDH and lead to toxicity. Therefore, a competitive inhibition with IMP is the least desirable, necessitating identification of mechanism for bacterial IMPDH inhibition.

Although C91 (1), a benzimidazole based inhibitor is one of the most potent HPIMPDH inhibitor known, the benzimidazole scaffold suffers from limitations due to its poor metabolic profile ${ }^{13}$. With the aim of identifying new selective inhibitors of this bacterial IMPDH, we synthesized several indole-based small molecules. Indole based molecules have previously been identified as inhibitors of human IMPDH but with different structural profile $^{14}$. The molecules studied here were designed to avoid any inhibition of the host IMPDH. These molecules were investigated for their inhibitory potential against $H$ IMPDH and Homo sapiens IMPDH (HsIMPDH). From the inhibitory studies, we found that these small molecule inhibitors have a good inhibitory potential with selectivity over host IMPDH. Further, we have taken efforts to study the mechanism of $H p$ IMPDH inhibition with selected benzimidazole and indole-based small molecules (Fig. 1).

We found that the known $H p$ IMPDH inhibitor $\mathbf{1}^{6}$, a benzimidazole-based small molecule, is an uncompetitive IMPDH inhibitor with respect to both the substrates IMP and $\mathrm{NAD}^{+}$. Interestingly the indole-based small molecule 2 that are carefully designed and synthesized found to be noncompetitive inhibition towards $H p I M P D H$ versus both IMP and NAD ${ }^{+}$.

These findings indicated that the novel indole-based compound binds to different complexes of IMPDH and its substrates. This would be beneficial to design new and selective inhibitors of HPIMPDH, opening doors for developing highly selective inhibitors of $H$ IMPDH. Since competitive IMPDH inhibitors with respect to IMP binding site lack selectivity over host, uncompetitive and non-competitive inhibitors are much preferable. Notably in case of such inhibitors, increase in the substrate concentration does not affect the affinity of the inhibitors ${ }^{15}$.

\section{Results and Discussion}

Cloning, Expression, Purification and Characterization of $H$ pIMPDH. The recombinant $H p I M P D H$ was expressed in Rosetta ${ }^{\mathrm{TM}}$ (DE3) pLysS using pET-28a $(+)$ expression vector. The protein was purified using affinity chromatography (Ni-NTA) and size exclusion chromatography. Further, the recombinant $H p I M P D H$ was confirmed by western blot using anti His-tag antibody (Fig. 2). The final concentration of the protein was found to be $3 \mathrm{mg} / \mathrm{mL}$ (total volume of $1 \mathrm{~mL}$ ) from one-liter culture. The characterisation of recombinant $H p \mathrm{IMPDH}$ by LC-MS/MS resulted in $65 \%$ sequence match using peptide mass fingerprint analysis between recombinant $H p I M P D H$ and the $H$ IMPDH protein sequence obtained from the database.

The secondary structure prediction of purified recombinant $H p I M P D H$ is analyzed via Circular Dichroism (CD) (Fig. 3). A negative peak at $208 \mathrm{~nm}$ and $222 \mathrm{~nm}$ in the spectra indicated presence of alpha helices and a positive peak at $196 \mathrm{~nm}$ suggested presence of beta sheets ${ }^{16,17}$. The quantification of the secondary structure elements ${ }^{18,19}$ showed that $H$ PIMPDH comprises of $37 \%$ alpha helices, $17 \%$ of beta strand, $7 \%$ turns and $21 \%$ others(loops). The HPIMPDH secondary structure results obtained from CD were compared with the secondary structures of other Pro-IMPDHs and Euk-IMPDHs (Table S5). The reported CD spectra ${ }^{20}$ and crystal structure of other IMPDHs showed the presence of $22-34 \%$ alpha helices and $9-29 \%$ of beta sheet ${ }^{21}$. Hence, this confirms the secondary structure of $H$ PIMPDH found to be in correlation with the other reported IMPDHs.

Enzymatic activity and kinetics. Before carrying out enzymatic assays, it is important to determine the enzyme activity. After confirming the activity, the recombinant $H p \mathrm{IMPDH}(100 \mathrm{nM})$ was utilized for the enzyme kinetic studies. The studies were performed by varying IMP concentrations while keeping $\mathrm{NAD}^{+}$concentration $(600 \mu \mathrm{M})$ constant and by varying $\mathrm{NAD}^{+}$concentration while keeping IMP concentration $(500 \mu \mathrm{M})$ constant to study the kinetics of $H$ IMPDH for substrate (IMP) as well as for cofactor $\left(\mathrm{NAD}^{+}\right)$. As reported earlier by Gollapalli et al., HpIMPDH follows the Michaelis-Menten type of kinetics for both IMP and NAD ${ }^{+}$, the plots of initial velocity versus NADH concentration were found to be hyperbolic ${ }^{6}$ (Fig. 4). The $\mathrm{K}_{\mathrm{M}}$ values of $H p$ IMPDH for IMP and $\mathrm{NAD}^{+}$were found to be $19.38 \pm 1.53 \mu \mathrm{M}$ and $76.37 \pm 1.09 \mu \mathrm{M}$ respectively. In addition, the $\mathrm{k}_{\text {cat }}$ values 


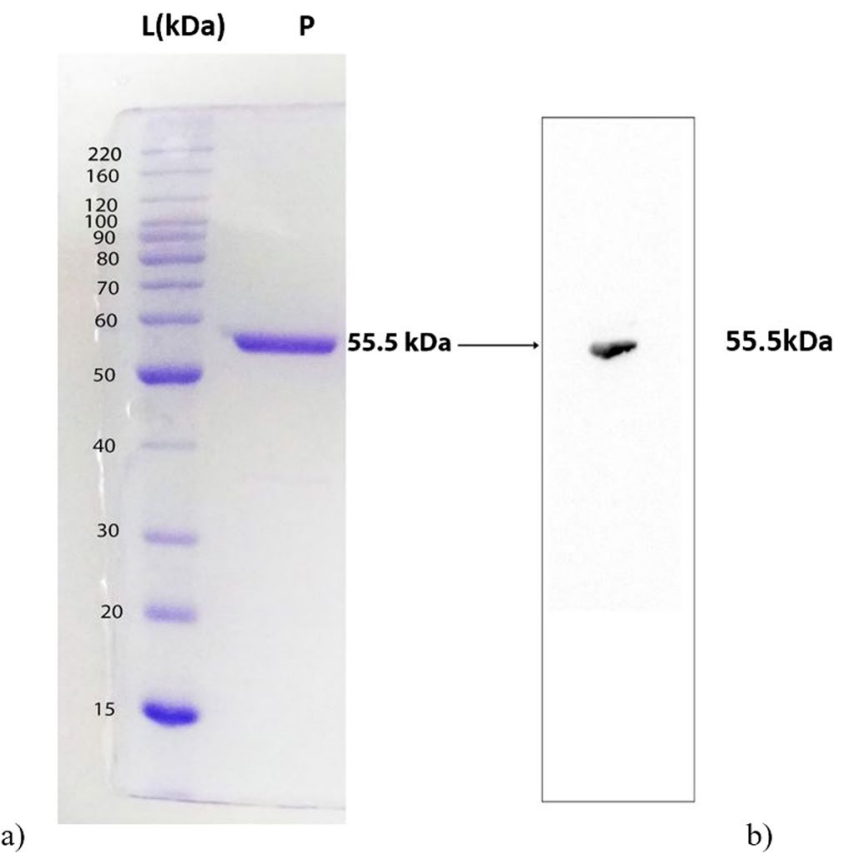

Figure 2. (a) SDS-PAGE gel; L-Ladder and P-Purified HpIMPDH protein (b) Western blot result of purified recombinant $H p I M P D H$. Full-length blots/gels are presented in Supplementary Fig. S15.

\section{Spectrum of HpIMPDH}

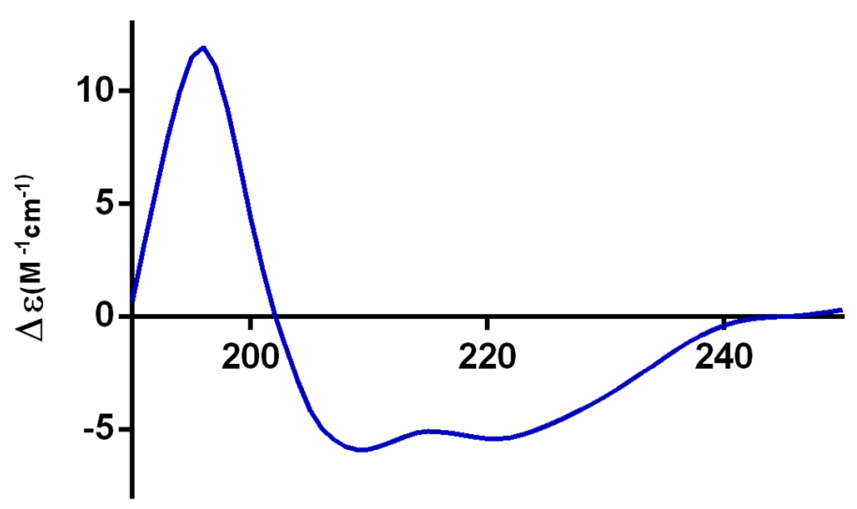

Wavelength $(\mathrm{nm})$

Figure 3. CD spectrum of purified HpIMPDH indicating the presence of alpha helix (negative peak at 208 and $222 \mathrm{~nm}$ ) and beta strand (positive peak at $196 \mathrm{~nm}$ ).
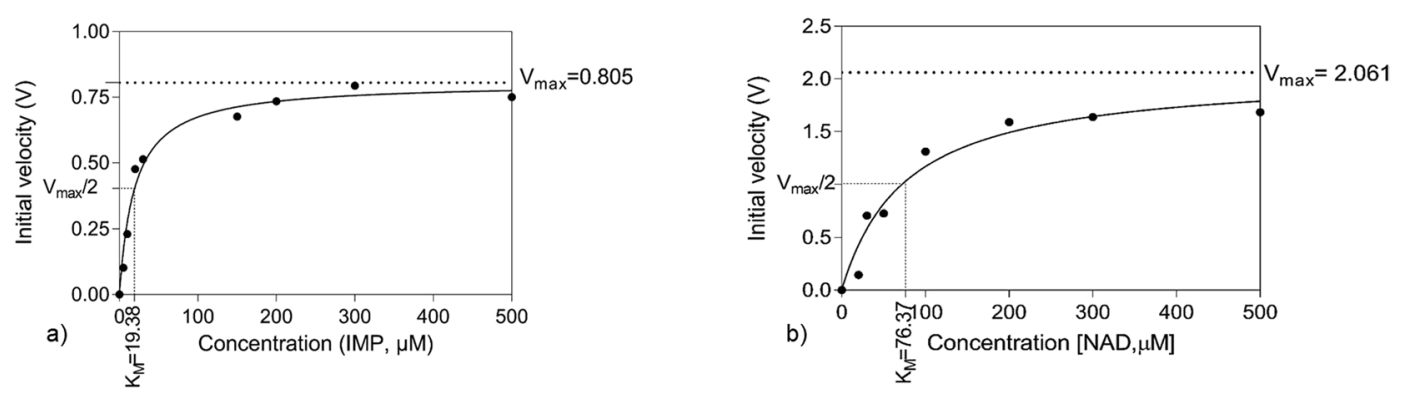

Figure 4. Enzyme activity of $H p I M P D H$ was determined in the presence of (a) Varying concentrations of IMP and (b) Varying concentrations of $\mathrm{NAD}^{+}$. The curves were fitted using the Michaelis-Menten equation. 


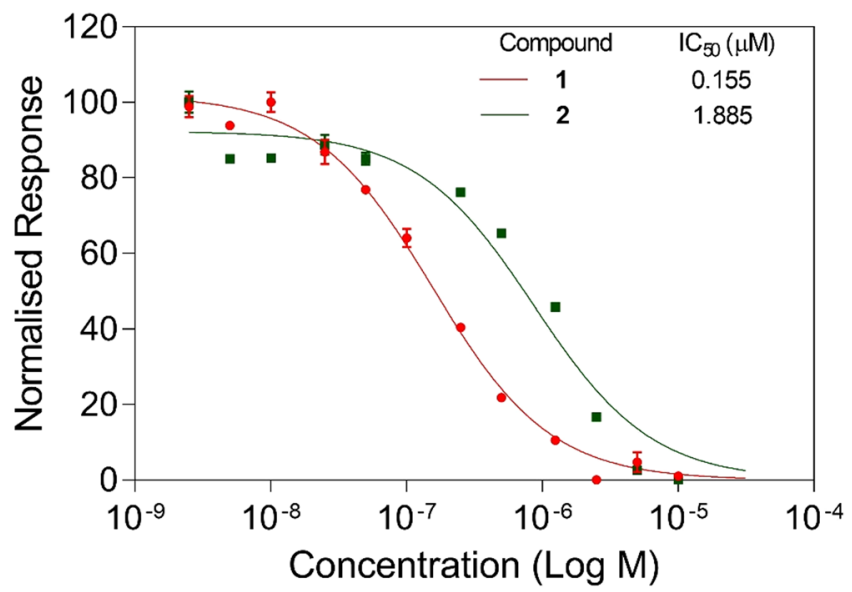

Figure 5. Concentration response curve for the inhibitory effect of $\mathbf{1}(\mathbf{C 9 1})$ and $\mathbf{2}$ against HpIMPDH. The data were fitted using 4 parameter Hill equation and each data point represents mean of triplicate.

\begin{tabular}{|l|l|}
\hline Compounds & $\mathrm{IC}_{50}(\boldsymbol{\mu M})$ \\
\hline 1 & $0.155 \pm 0.082$ \\
\hline 2 & $1.885 \pm 0.42$ \\
\hline
\end{tabular}

Table 1. HPIMPDH inhibition data for compounds $\mathbf{1}$ and 2.

of the enzyme for the substrates IMP and $\mathrm{NAD}^{+}$were found to be $5.57 \pm 0.88\left(\mathrm{~S}^{-1}\right)$ and $5.96 \pm 0.53\left(\mathrm{~S}^{-1}\right)$ respectively. The kinetics of HPIMPDH was then compared with other Pro-IMPDHs and Euk-IMPDHs (Table S6), which showed the broad differences of values among them. The $\mathrm{K}_{\mathrm{M}}$ (IMP) of $H p I M P D H$ was comparable with the $C$ IMPDH and BPIMPDH (Pro-IMPDH).

Enzyme inhibition and mechanistic studies. The inhibitory effect of the newly synthesised indole-based molecule (2) and selected benzimidazole small molecule (1) against HpIMPDH is investigated by monitoring the change in the initial velocity of $\mathrm{NADH}$ formation. To calculate the $\mathrm{IC}_{50}$ values, the initial velocity of the reaction is plotted against concentrations of the small molecule inhibitor, and the data is fitted with the Hill equation. A representative concentration-response curve for compound $\mathbf{1}$ and $\mathbf{2}$ are shown in Fig. 5.

The $\mathrm{IC}_{50}$ value of selected benzimidazole and indole-based small molecules are given in Tables 1 and S3. The indole-based molecules when screened at $10 \mu \mathrm{M}$ concentration showed no substantial effect on the enzyme activity of human IMPDH-II (Fig. S8). This suggests the selectivity of the indole-based molecules towards the HPIMPDH.

There are three important mechanisms of enzyme inhibition, viz. competitive, non-competitive and uncompetitive inhibition. The IMP binding site is highly conserved in all the IMPDHs. Hence, any inhibitor that shows competitive inhibition against IMP would lose selectivity over host IMPDH and subsequently would lead to undesirable side effects. Therefore, to find out the type of inhibition by the selected small molecule inhibitors, kinetic studies were performed in the presence of several concentrations of the inhibitor and varying concentration of IMP or NAD ${ }^{+}$. The initial velocity data were plotted using Lineweaver-Burk equation using GraphPad Prism.

As in Fig. 6: Ia and Ib, compound 1 inhibited HpIMPDH in an un-competitive manner against both IMP and $\mathrm{NAD}^{+}$. The $\mathrm{K}_{\mathrm{M}}$ and $\mathrm{V}_{\max }$ both found to be reduced with an increase in the inhibitor concentration, suggesting binding of the inhibitor to the substrate-enzyme complex. This important finding stresses the selectivity of the molecule towards bacterial IMPDH over human IMPDH. In contrast, IMP analogues have previously been shown to be competitive inhibitors against IMP and noncompetitive inhibitors against $\mathrm{NAD}^{+}$. The uncompetitive behaviour of 1 towards both IMP and $\mathrm{NAD}^{+}$suggests that it has a strong preference for binding to the E-XMP* complex ${ }^{15}$. This will be highly advantageous in-vivo as increased substrate concentration would lead to increased inhibition of the IMPDH unlike competitive behavior. First generation $\mathrm{C}$ series inhibitors have been found to be the non-competitive inhibitors ${ }^{6}$, whereas the second-generation 1 molecule ${ }^{12}$ showed uncompetitive inhibition. Mycophenolic acid (MPA), one of the potent inhibitor of HsIMPDH (both I and II) has also shown a similar inhibition mechanism against both IMP and NAD ${ }^{+22}$.

In the current study, we have also investigated new indole-based molecules for their ability to inhibit $H P I M P D H$ (inhibition data given in supplementary information). In the inhibitory studies, it was observed that compound 2 was able to inhibit $H p I M P D H$ with an $\mathrm{IC}_{50}$ value in the micromolar range. The kinetic studies carried out with compound 2 showed inhibitory behaviour different from that of the C91. As can be seen from Fig. 6-IIa and IIb, compound 2 showed a typical Lineweaver Burk plot for non-competitive inhibition against both IMP and $\mathrm{NAD}^{+}$, where regression lines meet on the X-axis. Here, the $\mathrm{K}_{\mathrm{M}}$ values of the $H p \mathrm{IMPDH}$ remained 
la)

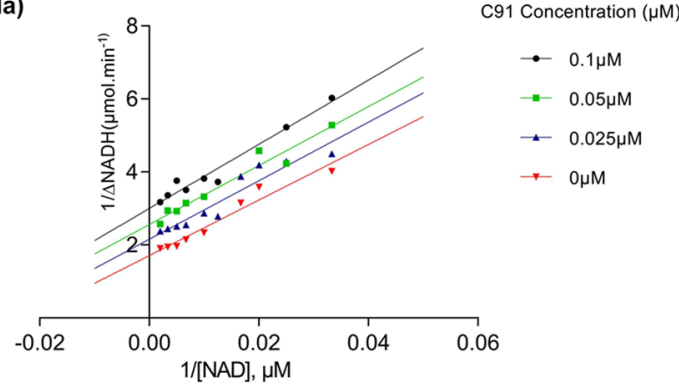

Ib)

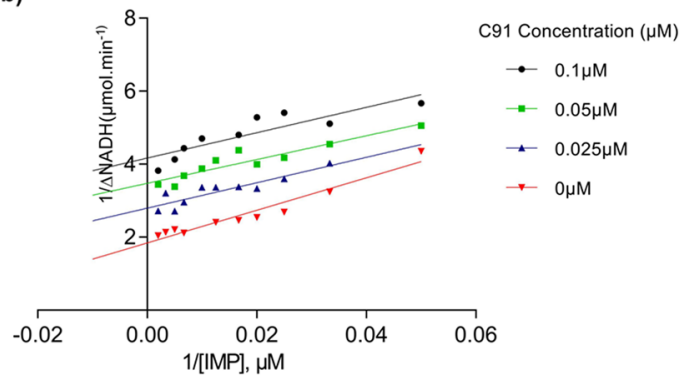<smiles>O=C(Cn1c(-c2ccccn2)nc2ccccc21)Nc1cccc2ccccc12</smiles>

1

Ila)
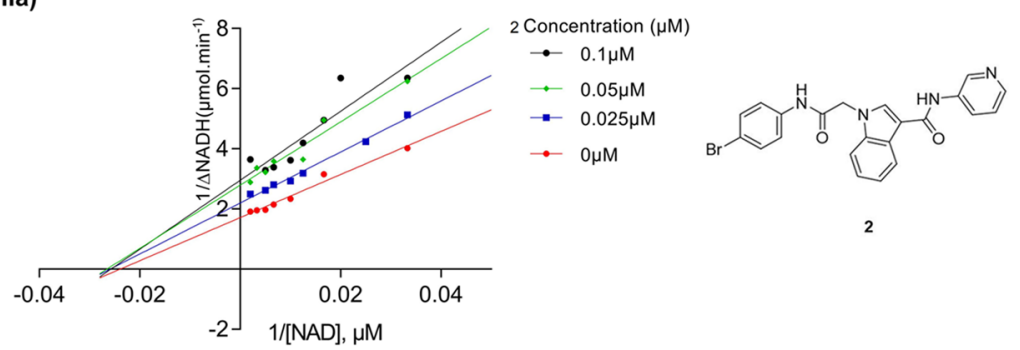

Illb)

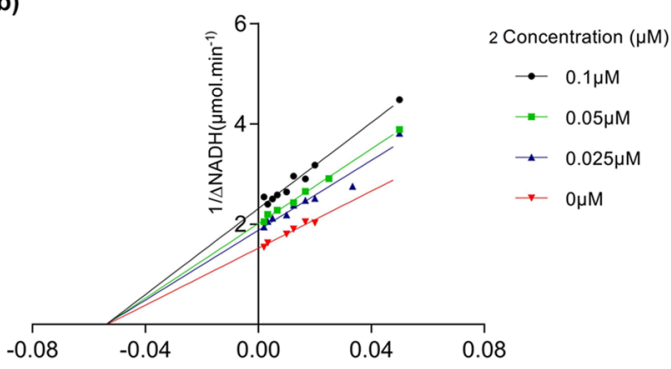

Figure 6. Mechanism of HPIMPDH inhibition by small molecules 1 (C91) and 2 was studied against varying concentrations of IMP and $\mathrm{NAD}^{+}$. The data is plotted using the Lineweaver-Burk equation.

constant since the inhibitor does not bind to the active site. In addition, there was a decrease in the $V_{\max }$ with the increasing concentration of inhibitor due to the low functional enzyme concentration.

In the case of non-competitive inhibition, the inhibitor binds to an allosteric site and changes the conformation of the active site. This type of inhibition is independent of substrate concentration as the inhibitor has the ability to bind and inhibit the free enzyme as well as the enzyme-substrate complex (unlike uncompetitive inhibitors that can inhibit only enzyme-substrate complexes). This suggests a preference of the inhibitor molecule 2 for the E•IMP and E-XMP* complexes, making the enzyme incapable of catalysing IMP to XMP conversion due to conformational changes in the IMP and $\mathrm{NAD}^{+}$binding sites. The same kind of mechanism has been observed for known IMPDH inhibitors TAD (thiazole-4-carboxamide adenine dinucleotide) and SAD (selenium analogue) ${ }^{23}$.

Multiple Sequence Alignment (MSA) of HpIMPDH with IMPDHs of other organisms. MSA is performed to identify the suitable drug-binding site in the bacterial IMPDH enzyme that affects specificity over host IMPDH. MSA of HPIMPDH with other Pro-IMPDHs and HsIMPDH-II is carried out. The protein sequences of $H p I M P D H$ taken for analysis is carfully studied with the reported crystal structures of IMPDH for designing small molecules. The result of MSA gave a clear assessment of conserved and non-conserved residues 


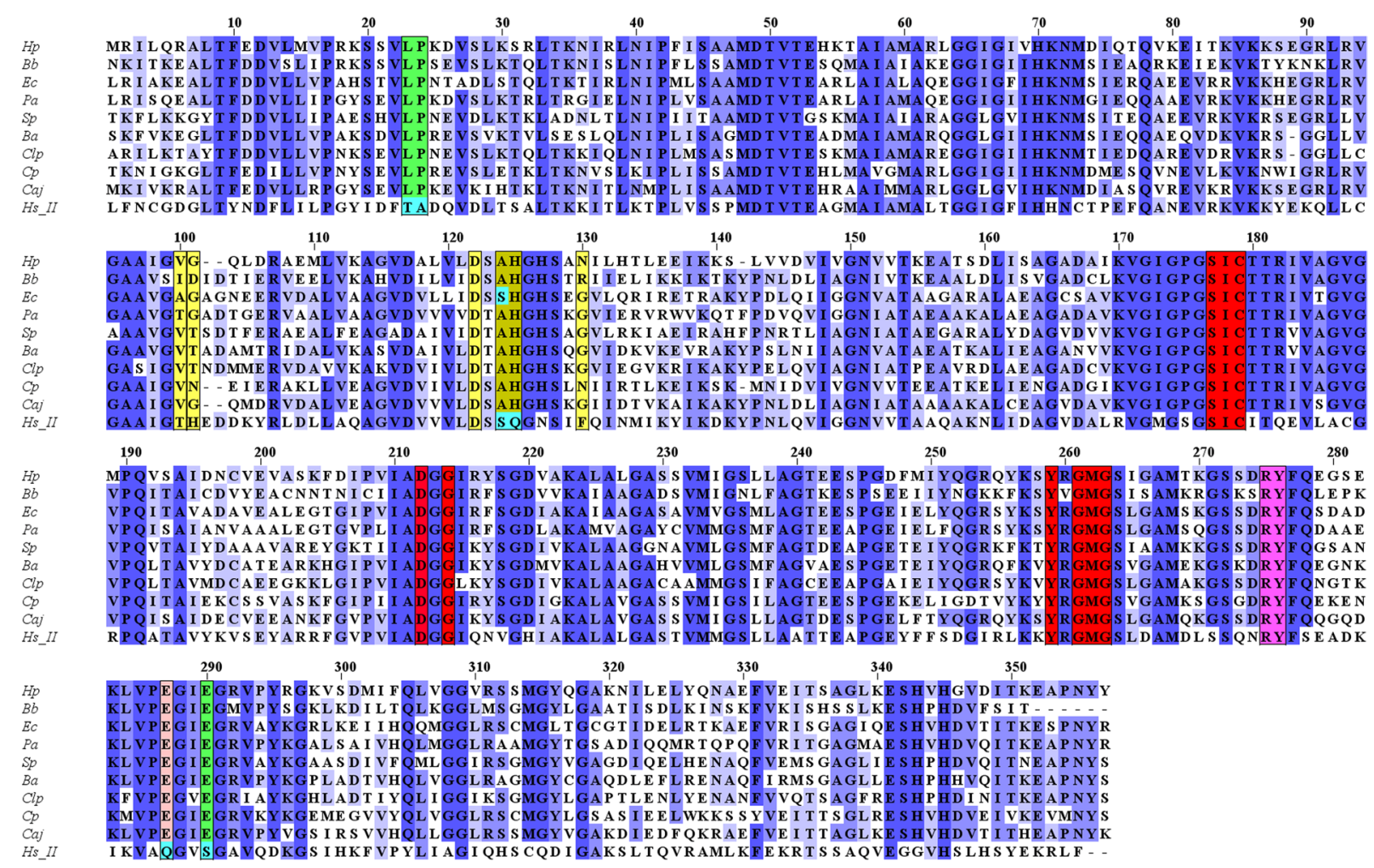

Figure 7. Multiple sequence alignment of various Pro-IMPDHs including HpIMPDH with HsIMPDH-II: Intensity of the purple colour indicates the percentage of the sequence conservation; Residues binding to C91 are coloured in green $(H p, B b, P a, S p . B a, C l p, C p, C a j)$ and $c y a n\left(E c, H s \_I I\right)$; Residues interacting with NAD $^{+}$ in $H s$ IMPDH-II and the corresponding residues in the Pro-IMPDHs are in indicated in yellow; Residues interacting with both 1 and $\mathrm{NAD}^{+}$are noted in greenish yellow; Residues interacting with IMP are indicated in red; Conserved residues RY in the flap region is indicated in the magenta; Key residue binding to the Mycophenolic acid is coloured in cyan for HsIMPDH-II and the corresponding residues in the Pro-IMPDHs coloured in peach.

in HPIMPDH as compared to other IMPDHs. The residues involved in key interactions are (Fig. 7), are conserved among all IMPDHs ${ }^{15,24}$. The Fig. 7 shows the conserved residues among the Pro-IMPDHs with respect to $H$ IMPDH and the HsIMPDH-II. The intensity of the purple colour corresponds to the percentage conservation of the residues among the species. Among the Pro-IMPDHs, $H p I M P D H$ has a higher percentage of sequence similarity with Campylobacter jejuni IMPDH (CjIMPDH) and CPIMPDH.

The sequence comparison of HPIMPDH with Euk-IMPDHs has revealed that the key binding residue of Mycophenolic acid (HsIMPDH-II inhibitor) ${ }^{25}$ is different in Euk-IMPDHs (Gln400) and HpIMPDH (Glu419). However, these residues are conserved in their own class. Reported crystal structures of Bacillus anthracis IMPDH (BaIMPDH) [PDB ID: 4MY9], Clostridium perfringens (ClpIMPDH) [PDB ID: 4Q33] and CjIMPDH [PDB IDL $4 \mathrm{MZ8}$ ] with known inhibitor $\mathbf{1}^{26-28}$ suggest an interaction of the compound with Glu290 by a hydrogen bond and appears to be a key interaction between Pro-IMPDH and 1. This Glu290 residue is conserved over Pro-IMPDH but it is replaced by Ser290 in HsIMPDH-II suggesting species-specific inhibitor of IMPDH (Fig. 7). Other residues reported to be involved in interaction with 1 via hydrophobic interactions are Leu23, Pro24, Ala125, His126, Met268. Except Met268 other residues are not conserved in Pro-IMPDHs and HsIMPDH-II. 1 effectively interacts only with two of the amino acid residues (Ala125 and His126) that bind to NAD ${ }^{+}$through hydrophobic interactions. Leu23, Pro24, Met268and Glu290 are other amino acids which are not a part of either IMP or NAD ${ }^{+}$ binding site.

MSA results suggested that $\mathbf{1}$ is not a competitive inhibitor and these observations agreed with our experimental results. The residues, 280-340 (Fig. 7), form a part of the flap region of the active site binds to $\mathrm{NAD}^{+}$. This region is highly divergent in HsIMPDH-II and Pro-IMPDHs which make this region a suitable target to design species-specific inhibitors ${ }^{6,15,29,30}$.

Prediction of the binding site. To analyse the binding site of the inhibitors in the protein, the $3 \mathrm{D}$ - structure of HpIMPDH has been generated from S. Pyorgenes (PDB ID: 1ZFJ) by SWISS-MODEL ${ }^{31}$ and validated. The generated structure was utilised to examine the mode of binding of non-competitive and uncompetitive inhibitors to the $H p I M P D H$ in an attempt to rationalise its activity against $H p I M P D H .1$ was found to be binding to the protein with the best ranking and docking score of $-5.3 \mathrm{kcal} \mathrm{mol}^{-1}$. The binding mode of $\mathbf{1}$ is similar to the way C64 (C series CPIMPDH inhibitor) binds to the $C \mathrm{PIMPDH}^{6}$. The naphthyl ring of 1 was found to be involved in 

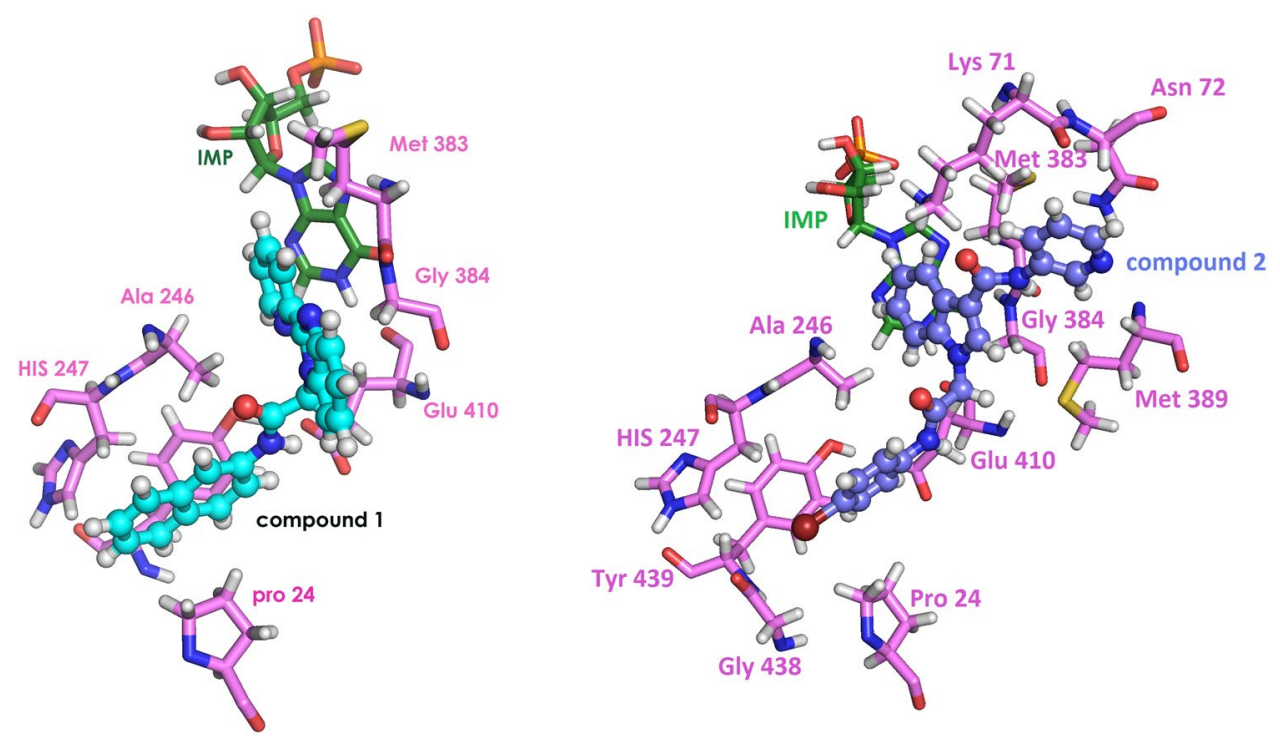

Figure 8. Interactions of compounds 1 and 2 at the binding site of $H p I M P D H$.

$\pi-\pi$ interaction with His 247 and Pro 24. The pyridyl ring forms a hydrophobic interaction with hypoxanthine ring of IMP and Met 383 (Fig. 8).

2 (non-competitive inhibitor) was found to be binding to the protein with the docking score of $-6.9 \mathrm{kcal} \mathrm{mol}^{-1}$. The 4-bromo phenyl group of the compound was found to be stabilized by hydrophobic interactions with residues His 247, Tyr 439, Gly 438 and Pro 24. Similar binding was reported in the co-crystal of TfIMPDH with thiazole-4-carboxamide adenine dinucleotide (TAD) ${ }^{32}$. Further, 2 was stabilized by hydrogen bonding with Lys 71 and Met 383. In 2 the N-phenyl-1H-indole-3-carboxamide moiety makes different interaction with IMPDH and orients into top right hydrophobic region. These strong interactions of $\mathbf{2}$ at the active site can be reasoned for its activity towards $H$ PIMPDH (Fig. 8)

\section{Conclusion}

Bacterial IMPDH, a vital enzyme in the nucleotide biosynthesis pathway has recently received a lot of attention as a possible target to treat multi-drug resistant infections. In the current work, the recombinant $H p I M P D H$ kinetic profile was characterized and it showed kinetic behavior of bacterial IMPDH and distinct from that of $H s I M P D H-I$ and II. These results suggest the possibility of selective inhibition of the HPIMPDH. We are reporting a new indole-based scaffold to target the $H p I M P D H$ that shows a good inhibition against $H p I M P D H$ and not against HsIMPDH-II confirming its selectivity over the host IMPDH. Benzimidazole based molecules have limited use due to their bad metabolite profile that could be overcome with development of indole based small molecule inhibitors of bacterial IMPDH.

In the current study, we have also studied the mechanism of inhibition of selected inhibitors for both the substrates, IMP and $\mathrm{NAD}^{+}$. From the results, it is clearly observed that the structural changes in the inhibitor influences not only the effectiveness $\left(\mathrm{IC}_{50}\right)$ of the inhibitor but also affects the affinity of the enzyme/enzyme-substrate/ enzyme-product complexes. A well-known inhibitor $\mathbf{C 9 1}$ bearing a benzimidazole scaffold showed an uncompetitive behavior against IMP as well as $\mathrm{NAD}^{+}$while newly synthesized indole-based compound (2) showed a noncompetitive inhibition. Both benzimidazole and indole-based inhibitors have the advantage that the inhibitory effect of these compounds will not be affected by the increase in the substrate concentration. This is in contrast to the competitive inhibitors, where bacteria can gain the resistance by producing the substrates in higher concentrations to overcome the effect of inhibitors. The findings from the current study are encouraging as we were able to identify new indole-based molecules for the IMPDH inhibition that could be the new starting point towards the quest of finding better bacterial IMPDH inhibitors to fight infections.

\section{Experimental Section}

Identification of HpIMPDH by Mass spectrometry - Peptide mapping. $\quad$ LC-MS/MS peptide mapping analysis was performed to confirm the expression of $H p I M P D H$ in $E$. coli. The purified protein sample was excised from the gel and tryptic digestion carried out for the LC-MS/MS analysis (Proteomics facility, MBU, IISc, Bangalore). The resultant data were examined for Peptide Mass Fingerprinting (PMF) using MASCOT software. The MASCOT search matched digested peptide sequence with the $H p \mathrm{IMPDH}$ protein sequences available in the protein database (NCBI and SwissProt). Validation of protein identification was done based on the number of matched peptides, the extent of protein sequence coverage and the score probability ${ }^{33}$ (Tables S1 and S2).

Secondary structure characterization by CD spectroscopy. To analyze the secondary structure content, recombinant $H$ IMPDH protein $(700 \mu \mathrm{g} / \mathrm{ml})$ in $20 \mathrm{mM}$ Tris-Cl and $50 \mathrm{mM} \mathrm{NaCl}$ buffer $(\mathrm{pH} 7.8)$ was used for CD spectra analysis. The analysis was carried out using Jasco spectropolarimeter equipped with a peltier-type temperature control system at a wavelength scan of $190 \mathrm{~nm}$ to $250 \mathrm{~nm}$. Far UV CD spectra were recorded in 
$0.5 \mathrm{~mm}$ quartz cuvette at $25^{\circ} \mathrm{C}$. A signal-averaged over at least 3 scans were collected. The spectrum was analyzed for secondary structure content using CDSSTR software ${ }^{18}$ which was included in Dichroweb tool ${ }^{19}$.

Enzymatic activity and steady state kinetics of $\mathrm{HpIMPDH}$. IMPDH enzyme catalyzes the oxidation of IMP to XMP with concurrent reduction of $\mathrm{NAD}^{+}$to NADH. The formation of NADH can be monitored by measuring absorbance at $340 \mathrm{~nm}$ or fluorescence at $440 \mathrm{~nm}$ when excited at $340 \mathrm{~nm}$. In presence of active IMPDH enzyme, there is an increase in the formation of $\mathrm{NADH}$ which could be correlated with the catalytic activity of the enzyme.

Kinetic studies of recombinant $\mathrm{HpIMPDH}$ were performed with an assay buffer containing $50 \mathrm{mM}$ Tris- $\mathrm{Cl}$ $\mathrm{pH} 8,100 \mathrm{mM} \mathrm{KCl}$ and $1 \mathrm{mM}$ dithiothreitol (DTT) at a final volume of $200 \mu \mathrm{L}$ using black96F well plate (Tarsons Products Pvt. Ltd., Kolkata, India). Assay was performed using $100 \mathrm{nM} \mathrm{HpIMPDH}$ in the presence of varying concentrations of IMP or NAD ${ }^{+}$. The production of NADH was monitored by measuring fluorescence at $440 \mathrm{~nm}$ when excited at $340 \mathrm{~nm}$ using PerkinElmer EnVision Multilabel Reader (Perkin Elmer, Inc., MA, USA).

For determination of Michaelis-Menten constant $\left(\mathrm{K}_{\mathrm{M}}\right)$, data of initial velocity at varying concentrations of IMP $(5 \mu \mathrm{M}$ to $500 \mu \mathrm{M})$ at fixed $\mathrm{NAD}^{+}$concentration $(600 \mu \mathrm{M})$ or varying concentrations of $\mathrm{NAD}^{+}(10 \mu \mathrm{M}$ to $500 \mu \mathrm{M})$ at fixed IMP concentration $(500 \mu \mathrm{M})$ were determined. The initial velocity data was fitted using Michaelis-Menten equation (1) with the help of GraphPad Prism to calculate enzyme kinetic parameters.

$$
\mathrm{V}=\frac{\mathrm{V} \max [\mathrm{S}]}{\mathrm{KM}+[\mathrm{S}]}
$$

where, $\mathrm{V}$ is velocity, $\mathrm{V}_{\max }$ is maximum velocity, [S] is substrate concentration, $\mathrm{K}_{\mathrm{M}}$ is Michaelis-Menten constant.

Enzyme inhibition assay. The inhibitory effects of the benzimidazole and indole-based small molecules are listed in Fig. 1. Similar to enzyme kinetic studies, these were also determined by monitoring NADH concentration through the fluorescence measurement. In the presence of IMPDH inhibitor, decreased catalytic activity of the enzyme reduces the formation of $\mathrm{NADH}$. To determine the $\mathrm{IC}_{50}$ of the small molecule inhibitors, varying concentration $(2.5 \mathrm{nM}$ to $10 \mu \mathrm{M})$ of compounds were incubated with $100 \mathrm{nM} \mathrm{HpIMPDH}$ in assay buffer containing $50 \mathrm{mM}$ Tris- $\mathrm{HCl}$ (pH 8.6), $100 \mathrm{mM} \mathrm{KCl}$ and $1 \mathrm{mM}$ dithiothreitol (DTT). The assay mixture was incubated for $10 \mathrm{~min}$ at $25^{\circ} \mathrm{C}$ and reaction was initiated by addition of NAD ${ }^{+}$and IMP with a final concentration of $300 \mu \mathrm{M}$ and $250 \mu \mathrm{M}$ respectively. The production of $\mathrm{NADH}$ was monitored by measuring fluorescence at $440 \mathrm{~nm}$ when excited at $340 \mathrm{~nm}$ using PerkinElmer EnVision Multilabel Reader (Perkin Elmer, Inc., MA, USA) at $37^{\circ} \mathrm{C}$. In these assays C91 was used as a positive control.

$\mathrm{IC}_{50}$ values for all compounds were calculated with the help of software GraphPad Prism. The equation applied was:

$$
Y=\text { Bottom }+\frac{(\text { Top }- \text { Bottom })}{1+10^{((\text {LogIC50-X)*HillSlope })}}
$$

Conservation analysis of $\mathrm{HpIMPDH}$ with other Prokaryotic and Eukaryotic IMPDHs. The amino acid sequences of some eukaryotic and prokaryotic IMPDH enzymes whose crystal structures ${ }^{11,24,29,34-36}$ are known were taken from the UniProt database ${ }^{37,38}$. These were namely, euk-IMPDH: Homo sapiens I (P20839), Homo sapiens II (P12268), Ashbya gossypii (Q756Z6), Cricetulus griseus (G3I4W7); pro-IMPDH: Pseudomonas aeruginosa (Q9HXM5), Streptococcus pyogenes (P0C0H6), Escherichia coli (P0ADG7), Borrelia burgdorferi (P49058) and Cryptosporidium parvum (Q8T6T2). Multiple sequence alignment of HpIMPDH with eukaryotic and prokaryotic sequences were checked separately using a software Clustal Omega ${ }^{39}$. For better alignment, few initial amino acid residues were removed from the sequences. The results of Clustal Omega were analysed using Jalview software for the conservative and non-conservative residues ${ }^{40}$.

Sequences retrieval and template selection for molecular model generation. The amino acid sequence of $H$ PIMPDH (ERM21512.1 IMP dehydrogenase) from $H$. pylori was retrieved from NCBI in FASTA format. This FASTA sequence was directly taken into Swiss model workspace for HPIMPDH structure prediction. SWISS-MODE workspace was used to build three-dimensional protein structure of $H p I M P D H$, using experimentally determined crystal structures of related family members as templates.

The target sequence was searched with BLAST and HHBlits ${ }^{41}$ for evolutionarily related structures matching the target sequence. Model generation of HPIMPDH was carried out based on the target-sequence alignment using ProMod3. Visualisation and analysis of the model were done using the PyMOL and Maestro programmes ${ }^{42}$.

Molecular Model evaluation. In this work, we have utilised PROCHEK ${ }^{43}$, ProSA ${ }^{44}, \mathrm{RMSD}$ and Ramachandran plot analysis to validate the final model. The PROCHECK online software was used to check the stereochemical quality of the protein by analysing residue by residue bond length, bond angles, torsional angles, chirality. ProSA online server was run to check the sequence to structure and validation of predicted 3-D structure. Ramachandran plot analysis was performed to visualise energetically allowed regions for backbone $\phi / \psi$ torsion angles.

Molecular Docking. In silico docking studies were conducted using the Glide module (XP) of Schrödinger Maestro v11.2 software ${ }^{42}$. Docking consists of four steps: Protein Preparation ${ }^{45}$, Ligand Preparation ${ }^{45}$, Receptor Grid Generation, and Ligand Docking. For each docked ligand the best-docked pose with lowest Glide score value was recorded and compared. 


\section{References}

1. Deen, N. S., Huang, S. J., Gong, L., Kwok, T. \& Devenish, R. J. The impact of autophagic processes on the intracellular fate of Helicobacter pylori: more tricks from an enigmatic pathogen? Autophagy 9, 639-652, https://doi.org/10.4161/auto.23782 (2013).

2. Thirumurthi, S. \& Graham, D. Y. Helicobacter pylori infection in India from a western perspective. The Indian journal of medical research 136, 549-562 (2012).

3. Wroblewski, L. E., Peek, R. M. Jr. \& Wilson, K. T. Helicobacter pylori and gastric cancer: factors that modulate disease risk. Clinical microbiology reviews 23, 713-739, https://doi.org/10.1128/CMR.00011-10 (2010).

4. Rimbara, E., Fischbach, L. A. \& Graham, D. Y. Optimal therapy for Helicobacter pylori infections. Nature reviews. Gastroenterology \& hepatology 8,79-88, https://doi.org/10.1038/nrgastro.2010.210 (2011).

5. Megraud, F. H pylori antibiotic resistance: prevalence, importance, and advances in testing. Gut 53, 1374-1384, https://doi. org/10.1136/gut.2003.022111 (2004).

6. Gollapalli, D. R. et al. Structural determinants of inhibitor selectivity in prokaryotic IMP dehydrogenases. Chemistry \& biology 17, 1084-1091, https://doi.org/10.1016/j.chembiol.2010.07.014 (2010).

7. Cox, J. A. et al. Novel inhibitors of Mycobacterium tuberculosis GuaB2 identified by a target based high-throughput phenotypic screen. Sci Rep 6, 38986, https://doi.org/10.1038/srep38986 (2016).

8. Abrahamsen, M. S. et al. Complete genome sequence of the apicomplexan, Cryptosporidium parvum. Science 304, 441-445, https:// doi.org/10.1126/science.1094786 (2004).

9. Jackson, R. C., Weber, G. \& Morris, H. P. IMP dehydrogenase, an enzyme linked with proliferation and malignancy. Nature 256, 331-333 (1975).

10. Zhang, R. et al. Differential signatures of bacterial and mammalian IMP dehydrogenase enzymes. Current medicinal chemistry $\mathbf{6}$, 537-543 (1999).

11. Macpherson, I. S. et al. The structural basis of Cryptosporidium -specific IMP dehydrogenase inhibitor selectivity. Journal of the American Chemical Society 132, 1230-1231, https://doi.org/10.1021/ja909947a (2010).

12. Kirubakaran, S. et al. Structure-activity relationship study of selective benzimidazole-based inhibitors of Cryptosporidium parvum IMPDH. Bioorganic \& medicinal chemistry letters 22, 1985-1988, https://doi.org/10.1016/j.bmcl.2012.01.029 (2012).

13. Hedstrom, L., Liechti, G., Goldberg, J. B. \& Gollapalli, D. R. The Antibiotic Potential of Prokaryotic IMP Dehydrogenase Inhibitors. Current Medicinal Chemistry 18, 1909-1918, https://doi.org/10.2174/092986711795590129 (2011).

14. Beevers, R. E. et al. Novel indole inhibitors of IMPDH from fragments: Synthesis and initial structure-activity relationships. Bioorganic \& Medicinal Chemistry Letters 16, 2539-2542, https://doi.org/10.1016/j.bmcl.2006.01.090 (2006).

15. Hedstrom, L. IMP dehydrogenase: structure, mechanism, and inhibition. Chemical reviews 109, 2903-2928, https://doi.org/10.1021/ cr900021w (2009).

16. Kelly, S. M., Jess, T. J. \& Price, N. C. How to study proteins by circular dichroism. Biochimica et biophysica acta 1751, 119-139, https://doi.org/10.1016/j.bbapap.2005.06.005 (2005).

17. Greenfield, N. J. Using circular dichroism spectra to estimate protein secondary structure. Nat Protoc 1, 2876-2890, https://doi. org/10.1038/nprot.2006.202 (2006).

18. Sreerama, N. \& Woody, R. W. Estimation of protein secondary structure from circular dichroism spectra: comparison of CONTIN, SELCON, and CDSSTR methods with an expanded reference set. Anal Biochem 287, 252-260, https://doi.org/10.1006/ abio.2000.4880 (2000).

19. Whitmore, L. \& Wallace, B. A. Protein secondary structure analyses from circular dichroism spectroscopy: methods and reference databases. Biopolymers 89, 392-400, https://doi.org/10.1002/bip.20853 (2008).

20. Alexandre, T., Raynal, B. \& Munier-Lehmann, H. Two classes of bacterial IMPDHs according to their quaternary structures and catalytic properties. PloS one 10, e0116578, https://doi.org/10.1371/journal.pone.0116578 (2015).

21. Carr, S. F., Papp, E., Wu, J. C. \& Natsumeda, Y. Characterization of human type I and type II IMP dehydrogenases. The Journal of biological chemistry 268, 27286-27290 (1993).

22. Hedstrom, L. IMP dehydrogenase: mechanism of action and inhibition. Current medicinal chemistry 6, 545-560 (1999).

23. Hedstrom, L. \& Wang, C. C. Mycophenolic acid and thiazole adenine dinucleotide inhibition of Tritrichomonas foetus inosine $5^{\prime}$-monophosphate dehydrogenase: implications on enzyme mechanism. Biochemistry 29, 849-854 (1990).

24. Sintchak, M. D. et al. Structure and mechanism of inosine monophosphate dehydrogenase in complex with the immunosuppressant mycophenolic acid. Cell 85, 921-930 (1996).

25. Digits, J. A. \& Hedstrom, L. Kinetic mechanism of Tritrichomonas foetus inosine $5^{\prime}$-monophosphate dehydrogenase. Biochemistry 38, 2295-2306, https://doi.org/10.1021/bi982305k (1999).

26. Kim, Y., Makowska-Grzyska, M., Gu, M., Anderson, W.F., Joachimiak, A. X-ray diffraction data for the crystal Structure of the Inosine $5^{\prime}$-monophosphate Dehydrogenase with an Internal Deletion of the CBS Domain from Bacillus anthracis str. Ames complexed with inhibitor C91 (4MY9). RSC PDB, https://doi.org/10.18430/M34MY9 (2013).

27. Maltseva, N. et al. Crystal Structure of Inosine $5^{\prime}$-monophosphate Dehydrogenase from Clostridium perfringens Complexed with IMP and C91. RSC PDB, https://doi.org/10.2210/pdb4q32/pdb (2014).

28. Kim, Y. et al. Csgid, Center for Structural Genomics of Infectious Diseases. Crystal Structure of the Inosine 5'-monophosphate Dehydrogenase, with an Internal Deletion of CBS Domain from Campylobacter jejuni complexed with inhibitor compound C91, https://doi.org/10.2210/pdb4mz8/pdb (2013).

29. Colby, T. D., Vanderveen, K., Strickler, M. D., Markham, G. D. \& Goldstein, B. M. Crystal structure of human type II inosine monophosphate dehydrogenase: implications for ligand binding and drug design. Proceedings of the National Academy of Sciences of the United States of America 96, 3531-3536 (1999).

30. Rostirolla, D. C. et al. D. S. Biochemical characterization of Mycobacterium tuberculosis IMP dehydrogenase: kinetic mechanism, metal activation and evidence of a cooperative system. RSC Advances 4, 26271-26287, https://doi.org/10.1039/C4RA02142H (2014).

31. Biasini, M. et al. SWISS-MODEL: modelling protein tertiary and quaternary structure using evolutionary information. Nucleic Acids Research 42, W252-W258, https://doi.org/10.1093/nar/gku340 (2014).

32. Gan, L., Petsko, G. A. \& Hedstrom, L. Crystal structure of a ternary complex of Tritrichomonas foetus inosine $5^{\prime}$-monophosphate dehydrogenase: NAD+orients the active site loop for catalysis. Biochemistry 41, 13309-13317 (2002).

33. Li, Q. et al. Identification of Novel Laminin- and Fibronectin-binding Proteins by Far-Western Blot: Capturing the Adhesins of Streptococcus suis Type 2. Frontiers in cellular and infection microbiology 5, 82, https://doi.org/10.3389/fcimb.2015.00082 (2015).

34. Zhang, R. et al. Characteristics and crystal structure of bacterial inosine-5'-monophosphate dehydrogenase. Biochemistry 38 , 4691-4700, https://doi.org/10.1021/bi982858v (1999).

35. Rao, V. A., Shepherd, S. M., Owen, R. \& Hunter, W. N. Structure of Pseudomonas aeruginosa inosine $5^{\prime}$-monophosphate dehydrogenase. Acta crystallographica. Section F, Structural biology and crystallization communications 69, 243-247, https://doi. org/10.1107/S1744309113002352 (2013).

36. McMillan, F. M. et al. Crystal structure at 2.4 A resolution of Borrelia burgdorferi inosine $5^{\prime}$-monophosphate dehydrogenase: evidence of a substrate-induced hinged-lid motion by loop 6. Biochemistry 39, 4533-4542 (2000).

37. UniProt, C. UniProt: a hub for protein information. Nucleic acids research 43, D204-212, https://doi.org/10.1093/nar/gku989 (2015).

38. Magrane, M. \& UniProt, C. UniProt Knowledgebase: a hub of integrated protein data. Database: the journal of biological databases and curation 2011, bar009, https://doi.org/10.1093/database/bar009 (2011). 
39. Sievers, F. et al. Fast, scalable generation of high-quality protein multiple sequence alignments using Clustal Omega. Molecular systems biology 7, 539, https://doi.org/10.1038/msb.2011.75 (2011).

40. Waterhouse, A. M., Procter, J. B., Martin, D. M., Clamp, M. \& Barton, G. J. Jalview Version 2-a multiple sequence alignment editor and analysis workbench. Bioinformatics 25, 1189-1191, https://doi.org/10.1093/bioinformatics/btp033 (2009).

41. Remmert, M., Biegert, A., Hauser, A. \& Soding, J. HHblits: lightning-fast iterative protein sequence searching by HMM-HMM alignment. Nat Methods 9, 173-175 (2011).

42. Friesner, R. A. et al. Extra Precision Glide: Docking and Scoring Incorporating a Model of Hydrophobic Enclosure for ProteinLigand Complexes. Journal of Medicinal Chemistry 49, 6177-6196, https://doi.org/10.1021/jm051256o (2006).

43. Laskowski, R. A., MacArthur, M. W., Moss, D. S. \& Thornton, J. M. PROCHECK: a program to check the stereochemical quality of protein structures. Journal of Applied Crystallography 26, 283-291, https://doi.org/10.1107/S0021889892009944 (1993).

44. Wiederstein, M. \& Sippl, M. J. ProSA-web: interactive web service for the recognition of errors in three-dimensional structures of proteins. Nucleic Acids Research 35, W407-W410, https://doi.org/10.1093/nar/gkm290 (2007).

45. Madhavi Sastry, G., Adzhigirey, M., Day, T., Annabhimoju, R. \& Sherman, W. Protein and ligand preparation: parameters, protocols, and influence on virtual screening enrichments. Journal of Computer-Aided Molecular Design 27, 221-234, https://doi.org/10.1007/ s10822-013-9644-8 (2013).

\section{Acknowledgements}

The authors would like to dedicate this work to Prof. P C Srinivasan, Retired Professor of Organic Chemistry, University of Madras. The authors (S.K. and V.T.) thank Science and engineering research Board (SERB), DSTRamanujam fellowship for S.K. for the funding. V.S. thanks DBT for RA fellowship. K.J. thanks IIT-Gandhinagar for the postdoctoral fellowship. The authors also thank the Government of Gujarat, Center for Biomedical engineering, IIT Gandhinagar for the support.

\section{Author Contributions}

V.T., S.K. conceptualised the idea and protocol. S.K. and K.J. designed the molecules and synthesised them. G.P. cloned and purified IMPDH and done the biophysical assays under the supervision of V.T. V.S. helped in optimising IMPDH cloning and purification. K.J. and G.P. performed all the biochemical assays under the supervision of V.T. and S.K. A.S. performed the docking experiments and validated the model of IMPDH along with V.T. S.R. helped in synthesizing small molecules reported here. Analysis of the data is done collectively by V.T., S.K., K.J. and G.P. Manuscript writing is done collectively by V.T., S.K., K.J and G.P.

\section{Additional Information}

Supplementary information accompanies this paper at https://doi.org/10.1038/s41598-018-37490-x.

Competing Interests: The authors declare no competing interests.

Publisher's note: Springer Nature remains neutral with regard to jurisdictional claims in published maps and institutional affiliations.

Open Access This article is licensed under a Creative Commons Attribution 4.0 International License, which permits use, sharing, adaptation, distribution and reproduction in any medium or format, as long as you give appropriate credit to the original author(s) and the source, provide a link to the Creative Commons license, and indicate if changes were made. The images or other third party material in this article are included in the article's Creative Commons license, unless indicated otherwise in a credit line to the material. If material is not included in the article's Creative Commons license and your intended use is not permitted by statutory regulation or exceeds the permitted use, you will need to obtain permission directly from the copyright holder. To view a copy of this license, visit http://creativecommons.org/licenses/by/4.0/.

(c) The Author(s) 2019 